\title{
Cuerpos peritoneales libres o ratones peritoneales
}

\author{
Peritoneal loose bodies or peritoneal mice
}

\author{
Mario Andrés González-Chávez, ${ }^{*}$ Marco Antonio Ascencio-Martínez, ${ }^{\ddagger}$ \\ Alberto Manuel González-Chávez, ${ }^{\S}$ Sandra Minerva García-Osogobio»
}

Palabras clave: Cuerpos peritoneales libres, apéndices epiplóicos, apéndices omentales, ratones peritoneales.

Keywords: Peritoneal loose body, epiploic appendages, omental appendices, peritoneal mice.

\footnotetext{
* Servicio de Cirugía. Fundación Clínica y Hospital Médica Sur. Humanitas Medical Group Coyoacán. Hospital Español de México, Ciudad de México. México.

₹ Servicio de Cirugía. Centro Médico ABC (American British Cowdray), Ciudad de México. México.

$\S$ Servicio de Cirugía. Hospital Español de México. Humanitas Medical Group Coyoacán, Ciudad de México. México. " Servicio de Cirugía. Fundación Clínica y Hospital Médica Sur. Humanitas Medical Group Coyoacán, Ciudad de México. México.
}

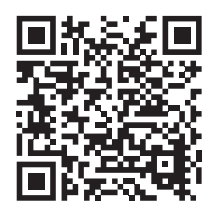

\section{RESUMEN}

Los cuerpos peritoneales libres son formaciones benignas generalmente descubiertas de manera incidental durante cirugía o autopsias. Es extremadamente raro encontrar cuerpos extraños libres en la cavidad peritoneal. Existe información limitada sobre la incidencia de cuerpos peritoneales libres en todo el mundo, solo se sabe que son más frecuentes en el sexo masculino con una relación hombre-mujer 18:4 y que en su mayoría se presentan entre los 50 y 70 años de edad debido principalmente a su baja incidencia y a que en su mayoría cursan asintomáticas. La teoría etiológica más aceptada propone que se originan a partir de apéndices epiplóicos torcidos e infartados que se desprenden de la serosa del colon y gradualmente se fibrosan y calcifican. Presentamos el caso clínico de un paciente de 81 años con apendicitis aguda en quien, como hallazgo incidental durante la laparotomía exploradora, se descubrieron tres cuerpos peritoneales libres. Fue un hallazgo incidental, inesperado, descubierto durante la laparotomía, sin relación alguna con la apendicitis aguda.

\section{ABSTRACT}

Free peritoneal bodies are benign formations generally discovered incidentally during surgery or autopsies. It's extremely rare to find free foreign bodies in the peritoneal cavity (there's limited information on the incidence of free peritoneal bodies worldwide, it's only known that they are more frequent in males with a male-female ratio 18:4 and than in most of them present between 50 and 70 years of age) mainly due to their low incidence and that they are mostly asymptomatic (the most accepted etiology theory proposes that they originate from twisted and infarcted epiploic appendages that detach from the serosa of the colon and gradually fibroses and calcifies. We present the clinical case of an 81-year-old patient with acute appendicitis in whom, as an incidental finding during exploratory laparotomy, three free peritoneal bodies were discovered (it was an incidental, unexpected finding, discovered during laparotomy, unrelated to acute appendicitis).

\section{INTRODUCCIÓN}

L os cuerpos peritoneales libres son formaciones cálcico-fibróticas encontradas generalmente de forma incidental en la cavidad abdominal, sin estar unidas o ser dependientes de algún órgano abdominal y sin contar con un aporte sanguíneo propio (de ahí su terminología "libres"). ${ }^{1}$ Existen diversas teorías sobre su etiología, siendo la más aceptada que se originan de apéndices epiplóicos, mismos que tras sufrir un proceso crónico de isquemia se desprenden a la cavidad peritoneal, con posterior acúmulo de capas superpuestas de albúmina y tejido fibrótico. ${ }^{1,2}$

Existe información limitada sobre la incidencia de cuerpos peritoneales libres en todo el mundo, debido - principalmente - a que son muy infrecuentes y a que en su mayoría corresponden a lesiones asintomáticas que pasan desapercibidas. Es aún más raro encontrar cuerpos peritoneales gigantes (mayores a $5 \mathrm{~cm}$ de diámetro) $)^{2}$ o múltiples de estos cuerpos, como es el caso del paciente que a continuación presentamos. En los ocho artículos que se revisaron, se reportan ocho casos de cuerpos

Citar como: González-Chávez MA, Ascencio-Martínez MA, González-Chávez AM, García-Osogobio SM. Cuerpos peritoneales libres o ratones peritoneales. Cir Gen. 2021; 43 (1): 36-39. https://dx.doi.org/10.35366/103912 
peritoneales únicos de entre 3 y $9.5 \mathrm{~cm}$ de diámetro, cinco de esos ocho cuerpos peritoneales libres reportados, eran mayores de $5 \mathrm{~cm}$ (gigantes), todos los pacientes descritos eran de sexo masculino de entre 50 y 72 años de edad. En uno de los artículos que revisamos, ${ }^{3}$ se presenta una tabla comparativa de 25 casos en total. El más antigüo de 1951, el más reciente de 2016. Cuatro mujeres (dos meses, 33, 33 y 69 años) y 21 hombres (de entre 47 y 79 años), 23 casos de cuerpo único y dos casos de múltiples cuerpos (un hombre de 63 años con dos cuerpos peritoneales libres de $5.8 \times 4.5 \times 3.7 \mathrm{~cm}$ y $5.2 \times$ $4.5 \times 3.7 \mathrm{~cm}$; y otro hombre de 79 años, con dos cuerpos de $7.0 \times 6.0 \mathrm{~cm}$ y $7.0 \times 6.0 \mathrm{~cm}$ ).

\section{PRESENTACIÓN DEL CASO CLÍNICO}

Hombre en la novena década de la vida, con dolor abdominal de 72 horas de evolución, con predominio a nivel de la fosa iliaca derecha (FID), asociado a episodio de bacteriemia, hiporexia, náusea, vómito y evacuaciones líquidas. Abdomen con dolor a la palpación en la FID, apendiculares positivos, peristalsis presente e hiperactiva, con datos de irritación peritoneal. Leucocitosis de $11.2 \times 10^{3} \mathrm{ul}$, con desviación a la izquierda: neutrofilia de $86.9 \%$. La tomografía abdomino-pélvica con medio de contraste intravenoso (Figura 1) mostró el apéndice cecal con datos de proceso inflamatorio agudo. Se realizó apendicectomía abierta por incisión tipo McBurney. Al ingresar a la cavidad abdominal se encontraron de manera

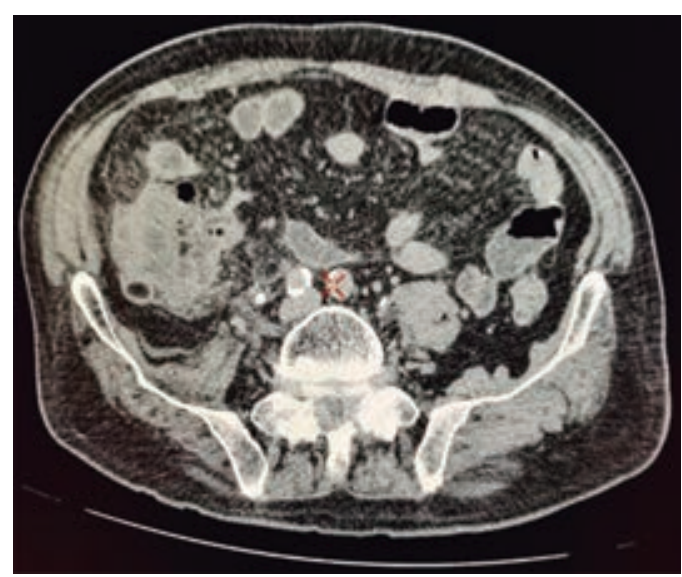

Figura 1: Tomografía abdominal con contraste IV.

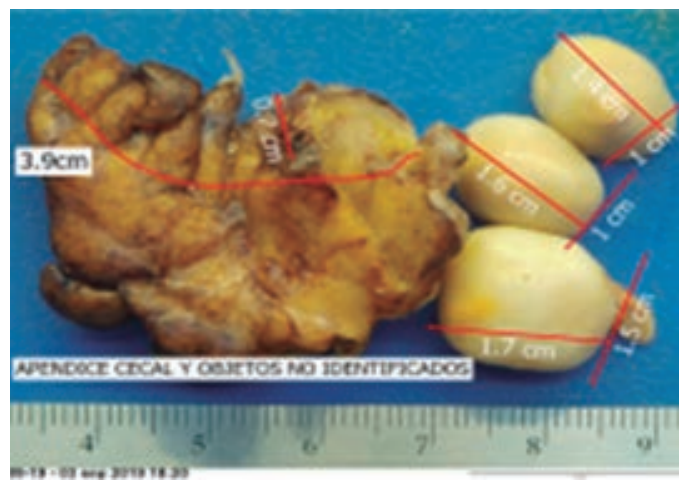

Figura 2: Objetos libres en cavidad abdominal y apéndice cecal.

incidental tres cuerpos peritoneales libres, los cuales se enviaron a estudio histopatológico, junto con el apéndice cecal resecado (Figura 2).

El análisis patológico macroscópico reportó tres neoformaciones ovoides de $17 \times 15 \times 10$ $\mathrm{mm}, 16 \times 10 \times 10 \mathrm{~mm}$ y $14 \times 10 \times 10 \mathrm{~mm}$ cada uno, de superficie lisa, amarilla y firme (Figura 3); al corte son sólidos, con una zona periférica gris blanquecina de $4 \mathrm{~mm}$ de espesor y una zona central amarillenta de consistencia firme (Figura 4).

La descripción microscópica consiste en apéndices epiplóicos con tejido conectivo denso en la periferia, con calcificación distrófica y zonas de necrosis grasa, sin otro tipo de alteraciones ni cambios histológicos de tipo neoplásico maligno. Apendicitis aguda fibrinopurulenta (Figura 5).

\section{DISCUSIÓN}

Las masas abdomino-pélvicas móviles o libres son extremadamente infrecuentes y por lo general tienden a localizarse en el hueco pélvico debido al efecto que produce la gravedad. ${ }^{2}$ Dentro del diagnóstico diferencial de masas móviles se debe tener siempre en mente la existencia de los cuerpos peritoneales libres. ${ }^{3}$ Los cuerpos peritoneales libres, también llamados por algunos autores "ratones peritoneales" o "migas peritoneales", 3 son formaciones cálcico-fibróticas que se encuentran libres en la cavidad peritoneal. Dado que en su mayoría son asintomáticas, en muchas ocasiones representan hallazgos incidentales durante cirugías o autopsias; ${ }^{1}$ en 
el caso de nuestro paciente también fueron asintomáticas, ya que no confirieron patología adicional al padecimiento apendicular. Solo en algunas ocasiones, dependiendo del tamaño y de su localización, pueden producir sintomatología poco específica caracterizada por dolor o molestia abdominal baja, estreñimiento, cuadros de obstrucción intestinal o síntomas urinarios como polaquiuria o retención aguda de orina. ${ }^{4}$ Existen muy pocos datos epidemiológicos publicados sobre estas neoformaciones; sin embargo, parece ser que son más frecuentes en el sexo masculino con una relación hombre-mujer 18:4 (lo cual coincide con el paciente que presentamos) y que en su mayoría se presentan entre los 50 y 70 años de edad, aunque existen reportes de su presencia desde los dos meses de edad. ${ }^{3}$ Los pocos reportes con los que se cuenta en la literatura son, en su mayoría, sobre cuerpos únicos gigantes mayores a $5 \mathrm{~cm}$ de diámetro, ${ }^{2-8}$ a diferencia del caso que presentamos en el que descubrimos no uno, sino tres diferentes cuerpos peritoneales, pero de menor tamaño que los de dichas publicaciones. En nuestro hospital (HMG Coyoacán), sin casos similares reportados desde 2016 a la fecha.

Se han propuesto diferentes teorías sobre el origen de estos cuerpos peritoneales libres, pero su etiología exacta es aún desconocida. Dentro de los posibles orígenes se incluyen apéndices epiplóicos, epiplón, anexos autoamputados o tejido adiposo pancreático; la teoría más aceptada es la que menciona que se originan de apéndices epiplóicos. ${ }^{3}$ El primero en describir los apéndices epiploicos fue Andre Vesalius, posteriormente diversos estudios anatómicos fueron realizados para describir otros aspectos anatómicos de los mismos. El primero en reportar una serie de casos de apéndices epiploicos

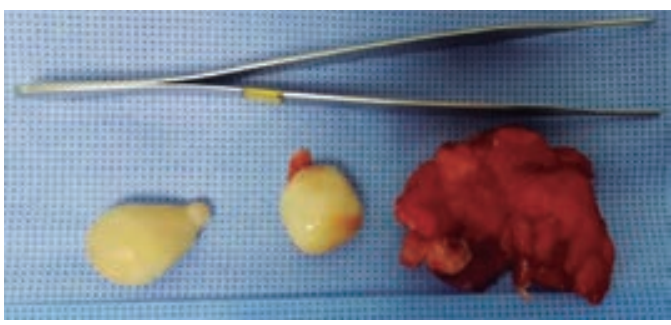

Figura 3: Apéndice cecal y objetos ovoides identificados en cavidad.

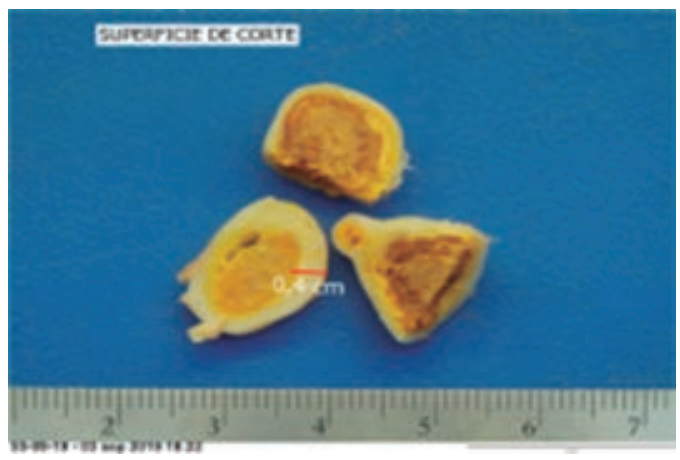

Figura 4: Aspecto macroscópico, superficie de corte.

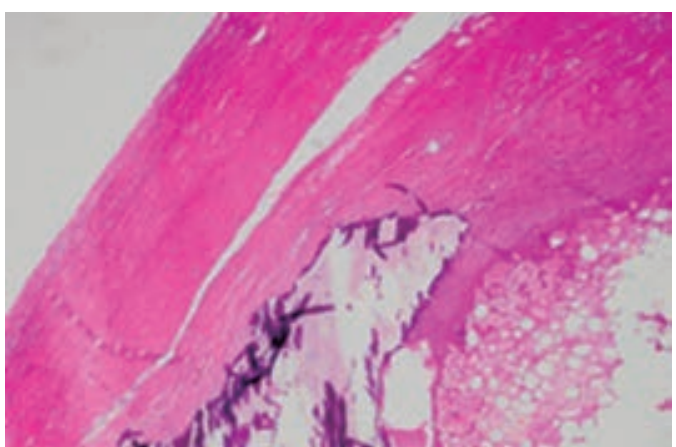

Figura 5: Pared fibrosa al análisis microscópico.

libres fue Harrigan en el año de $1917 .{ }^{5}$ La teoría sobre los cambios secuenciales que llevan a su formación fue presentada por Virchow en 1863, donde fue propuesto un aumento gradual y progresivo de tejido graso dentro de un apéndice epiplóico (por lo general en el contexto de obesidad) lleva a la obliteración y obstrucción de los vasos sanguíneos del pedículo; después se presenta un proceso de torsión, estrangulamiento y necrosis del apéndice epiplóico, hasta su amputación. ${ }^{2}$ Posteriormente, en 1933, Patterson propuso que la isquemia es el factor etiológico predominante en su fisiopatología y que es la causa que conlleva a infarto del apéndice epiploico. Es el factor etiológico dominante en la fisiopatología, y que es dicha isquemia la que lleva al infarto y la amputación. ${ }^{4}$ Una vez que el apéndice epiplóico queda flotando libre en la cavidad abdominal, sufre un proceso de saponificación y calcificación. ${ }^{6}$ Con el paso de los años, la reacción peritoneal sobre este cuerpo libre y la deposición a su alrededor de líquido peritoneal (fluido sérico exudativo rico 
en proteínas, principalmente albúmina) produce el progresivo incremento en su tamaño mediante adición de capas fibrosas periféricas. ${ }^{2,4}$ Esta teoría fue comprobada en 1968 por Donado y Kerr quienes tomaron grasa peri-uterina de cuyos y la colocaron en la cavidad peritoneal de dichos animales, generando típicos cuerpos peritoneales libres cada vez más grandes con el paso del tiempo. ${ }^{7}$ Una vez formados, establecer un diagnóstico preoperatorio correcto resulta difícil, y en muchas ocasiones se confunden con neoplasias y se resecan innecesariamente. Son lesiones raras y asintomáticas, pero en ocasiones pueden ser detectadas por estudios de imagen. En tomografía computarizada se observan como masas, usualmente únicas, de forma redonda u ovalada, bien definidas, con calcificación central, rodeadas por un tejido blando periférico. En resonancia magnética se observan como masas bien circunscritas, hipointensas en $\mathrm{T} 1$ y $\mathrm{T} 2$, pero con un área de hiperintensidad central en T1. Por ninguna modalidad de imagen la masa presenta reforzamiento a la administración de medios de contraste, dado que carecen de aporte vascular. ${ }^{1}$ Si se realizan estudios de imagen seriados o en diferentes posiciones (supina o prona), al compararlos se puede apreciar la movilidad de la masa, evidenciando ubicaciones variables dentro de la cavidad. ${ }^{1,7}$ Se debe realizar un adecuado diagnóstico diferencial con enfermedades benignas (leiomiomas, rabdomiomas, teratomas, fibromas), enfermedades malignas (cáncer colorrectal, cáncer de ovario, linfomas, metástasis), cálculos urinarios, biliares o apendiculares, quistes dermoides, granulomas tuberculosos, calcificación de ganglios linfáticos, cuerpos extraños, quistes hidatídicos, entre otros. ${ }^{3,7} \mathrm{Si}$ se detectan de forma incidental en cirugía, situación que ocurrió en nuestro caso, los cuerpos peritoneales libres flotan dentro de la cavidad peritoneal y tienen aspecto de una concreción blanca, dura y brillante que da la apariencia de un huevo cocido. ${ }^{1,8}$ La extirpación quirúrgica con subsecuente examen histológico puede confirmar definitivamente el diagnóstico en función de las características morfológicas. Dichos hallazgos patológicos consisten en un núcleo central de grasa necrótica calcificada, laminada por capas de tejido fibroso acelular hialinizado en la periferia, ${ }^{1,2}$ como lo evidenciamos en los cuerpos encontrados en nuestro paciente. De lograrse un adecuado diagnóstico preoperatorio, por lo general los cuerpos peritoneales libres no requieren tratamiento y se manejan sólo con vigilancia. ${ }^{2}$ Es esencial que los médicos conozcan esta entidad y sus características en estudios de imagen, así como su posibles diagnósticos diferenciales, para con un alto índice de sospecha poder establecer el diagnóstico correcto y evitar intervenciones innecesarias, dado que se trata de neoformaciones benignas que pueden ser manejadas mediante vigilancia.

\section{REFERENCIAS}

1. Gayer G, Petrovitch I. CT diagnosis of a large peritoneal loose body: a case report and review of the literature. Br J Radiol. 2011; 84: e83-85.

2. Rajbhandari M, Karmacharya A, Shrestha S. Pathological diagnosis of peritoneal loose body: a case report. J Pathol Nepal. 2013; 3: 512-514.

3. Kosam S, Kujur P, Mire V. Peritoneal Mice' A Peritoneal Loose Body in Pelvic Cavity of 70 Years Old Man, An Incidental Finding-A Case Report. Int J Sci Res (Raipur). 2017; 6: 2366-2369.

4. Hedawoo JB, Wagh A. Giant peritoneal loose body in a patient with haemorrhoids. Trop Gastroenterol. 2010; 31: 132-133.

5. Harrigan AH. Torsion and inflammation of the appendices epiploicae. Ann Surg. 1917; 66(4):467478. doi: 10.1097/00000658-191710000-00014

6. Elsner A, Walensi M, Fuenfschilling M, Rosenberg R, Mechera R. Symptomatic giant peritoneal loose body in the pelvic cavity: A case report. Int J Surg Case Rep. 2016; 21: 32-35

7. Donado KJ, Kerr JF. Peritoneal loose bodies. Aust N Z Surg. 1968; 37:403-406.

8. Sewkani A, Jain A, Maudar K, Varshney S. 'Boiled egg' in the peritoneal cavity-a giant peritoneal loose body in a 64-year-old man: a case report. J Med Case Rep. 2011; 5: 297. doi: 10.1186/1752-1947-5-297.

Consideraciones éticas: Los autores declaran que los procedimientos seguidos se conformaron a las normas de ética. En este artículo no aparecen datos de pacientes.

Financiamiento: Recursos propios.

Conflicto de intereses: Los autores declaran que no existe ningún conflicto de intereses.

\author{
Correspondencia: \\ Sandra Minerva García-Osogobio \\ Puente de piedra 150. Torre de \\ Hospitalización primer piso. \\ Clínica de enfermedades digestivas \\ Médica Sur, Delegación Tlalpan. C.P. 14050 \\ Ciudad de México. \\ E-mail: lapcolon@gmail.com
}

